\title{
DESIGN OF SECURITY SYSTEM BASED ON RASPBERRY-PI
}

\author{
MOHANAD ABDULHAMID ${ }^{1}$, SINGOEE SHESHAI ${ }^{2}$ \\ ${ }^{1}$ AL-Hikma University, Iraq, ${ }^{2}$ University of Nairobi, Kenya \\ E-mail: moh1hamid@yahoo.com, researcher12018@yahoo.com
}

\begin{abstract}
As a critical constituent of many associations, protection and security precedence, video surveillance has set up its importance and benefits numerous instances with the aid of imparting immediate supervising of possessions, people, surroundings and property. This paper deals with the diagram strategy of an embedded real-time surveillance gadget based totally on Raspberry-Pi single board computer (SBC) for intruder detection which is reinforcing technology of surveillance to supply fundamental security to our life and associated control and alert operations. The suggested safety solution is hinging on our novel integration of cameras and action detectors into application of web. Raspberry-Pi is operating and controlling action detectors and video cameras for far flung sensing and surveillance, streams live video and files it for future playback. Also, this paper is focusing on growing a surveillance machine that detects strangers and to response speedily through taking pictures and relaying photos to proprietor based totally wireless module. This Raspberry-Pi based clever surveillance machine presents the concept of monitoring a region in a far-flung area. The suggested solution offers a fee advantageous ubiquitous surveillance solution, environment friendly and convenient to implement. Furthermore, the paper presents the idea of motion detection and tracking using image processing. This type of technology is of great importance when it comes to surveillance and security. Live video streams therefore be used to show how objects can be detected then tracked. The detection and tracking process are based on pixel threshold.
\end{abstract}

Keywords: Embedded system, Raspberry-Pi, surveillance system, motion detection, tracking, video processing

\section{INTRODUCTION}

The demands on video surveillance systems are rapidly increasing in the present day. One of the first things people want to know about their surveillance system whether or not they can connect to it over the internet for remote viewing. In the past, security systems had to be monitored by a guard who is locked away in a room all day watching the monitors to make sure that nothing would happen. The other option is to come back and review the footage, but damage could have happened. Therefore, researchers and scientists had to come up with ways of overcoming that and thus improving security at large.

Commercial spaces, hospitals, universities, warehouses and casinos require video taking pictures systems which have the capability to alert and file beside stay video streaming of the intruder. The improvements in video surveillance generation have made it possible to view your far-flung protection digital camera from any netenabled PC or smartphone from everywhere within the world. This encompasses using closed circuit TV (CCTV) (digital video recorder (DVRs)) structures and internet protocol (IP) cameras. This generation is superb however its value of implementation has validated to be an obstacle particularly for a small home software.

Therefore, new innovative technology revolves around affordability of a product in terms of its cost and ease of implementation. The Raspberry-Pi crosses both criteria in that it is a cheap, effective computer which can be interfaced with other modules to realize systems with immense functionality. A lot can be done on it ranging from motor speed control, automatic lighting, virtual private network (VPN) server, security system etc.

The Raspberry-Pi microcomputer has the ability of imposing a value effective protection gadget for various packages. This modern arising technology associated with protection offers ease and safe surroundings for small houses. The various objectives of the system are to detect an intruder, take an image of the intruder and convey an alert message to the facility owner. In doing so it thus allows for remote monitoring of homes from anywhere in the world.

The system to be designed cannot wholly replace the role of CCTV and IP surveillance cameras especially in large commercial set ups but will make it easy for low income homeowners to monitor their homes at a very affordable price. In addition to the fact that the Raspberry-Pi board is cheap, the camera to be used in this case is relatively cheap compared to the others. The whole security system circuitry is simple and easy to implement.

Image processing is a term that shows the processing on photograph or video frame which is taken as an input and the result set of processing is may be a hard and fast of associated parameters of a photograph. The reason of picture processing is visualization that's to observe the items that are not seen. Evaluation of human motion is one of the maximum recent and popular research subjects in virtual image processing. In which the motion of human is the vital a part of human detection and movement analysis, the purpose is to discover the motions of human from the heritage photograph in a video collection. It is also including detection and monitoring. The technique of item monitoring is segmenting a place of interest from a video frames and 
preserving track of its movement and position. Numerous works on the topic of this paper may be found in literatures [1-5].

\section{DESIGN METHODOLOGY}

For an embedded real-time surveillance system to be utilized for effective monitoring and alerting, the system must have at least three functions. These functions are detection, image processing and alert mechanism. The Raspberry Pi based security system is thus composed of mainly two parts. These are: hardware design and software design.

\subsection{Hardware design}

The entire system modules consist of seven parts components namely:

1 - Pi Model B+ controller;

2 - PIR motion sensor;

3 - RJ45 Ethernet connector;

4 - Pi camera module;

5 - Micro-SD card;

6 - LED and 220 Ohms resistor;

7 - USB powered cable.

\subsubsection{Raspberry-Pi model B+}

This is the model that is chosen to implement the work in this paper. It has merits over other models in that it has increased number of universal serial bus (USB) ports and large number of general purpose input/output (GPIO) pins.

\subsubsection{Booting-up the Pi model}

Raspbian 'Wheezy' image is written into the 4GB Micro-SD card. This is the operating system chosen to run on the Pi because the operating system has been optimized and ported to the Raspberry-Pi Ashton Raggatt McDougall (ARM) architecture. The operating system has very good integration with the hardware and comes pre-loaded with a graphical user interface (GUI) and development tools.

After slotting in the Micro-SD card and connecting RJ45 Ethernet cable to the Pi and the personal computer with Putty software (Putty is a secure shell (SSH) client used to remotely access and control the $\mathrm{Pi}$ from computer running on Windows platform) the system is powered. Putty is then started, and the default static IP address of the $\mathrm{Pi}$ is typed into the host name field. While doing this, windows PC is set to manual IP configuration. This is to allow it to communicate with the Raspberry Pi.

\subsubsection{Setting-up internet connection on the Pi}

Internet is necessary so that the Pi can communicate over network protocols and thus allow for installation of necessary Python packages. The architecture in Figure 1 is used to achieve that.

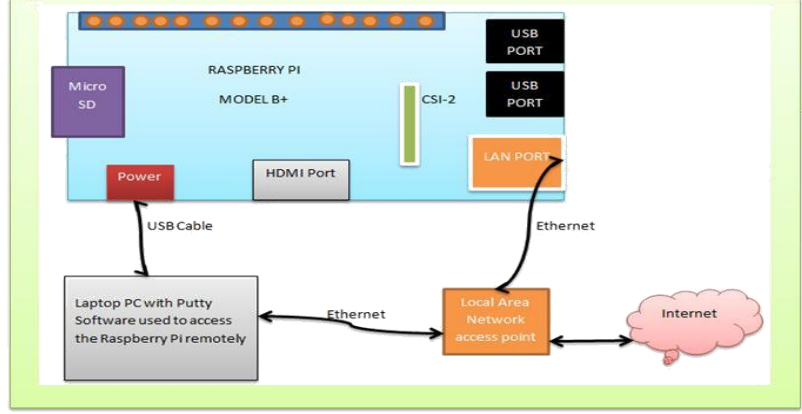

Figure 1. Internet set up architecture

Since the broadcast router uses dynamic host configuration protocol (DHCP) to dish out IP addresses to devices connected to it, it is necessary to change the IP address of the Pi from static to dynamic. This is done by editing the network interfaces file using the following command:

sudo nano /etc/network/interfaces

\subsubsection{Enabling the Pi camera}

This camera is made specifically for the Raspberry Pi. It is hooked to the Raspberry-Pi through camera serial interface (CSI-2) electrical port which is an extremely fast port. To configure and enable the camera, the following commands are executed at the command-line interface (CLI) of the Raspberry-Pi:

\begin{tabular}{|l|}
\hline sudo apt_get update \\
\hline sudo apt_get upgrade \\
\hline sudo raspi-config \\
\hline
\end{tabular}

After these configuration settings, the system is rebooted. This is done to ensure that the camera is allocated enough space in memory. The camera takes 5MP image and has a resolution of 1080 by 890 . And to ensure that the camera is well configured and functional, the following command is executed.

sudo raspistill -o image.png

By default, this command takes a three second image and save it in a file called image.png.

\subsubsection{Setting-up the passive infrared sensor}

This forms the prime motion sensor. It is used to control the entire system. The device used here is HC501SR passive infrared sensor. The detection range is 7 meters by 140 (degrees) coning angles. It has a delay time of 16 
seconds but adjustable. The ambient temperature is $253 \mathrm{~K}-323 \mathrm{~K}$. It is powered directly from the Pi through the $5 \mathrm{~V}$ dc supply pin. Its output is connected as the input to the programmable GPIO pin.

\subsubsection{Automatic light simulation}

Light-emitting-diode (LED) is used to simulate an automatic light control. This is designed to be controlled through the action of a passive infrared sensor (PIR) sensor. This device is connected to the GPIO pin through a $220 \Omega$ resistor.

\subsection{Hardware architecture}

The entire system modules are interfaced together as shown in Figure 2.

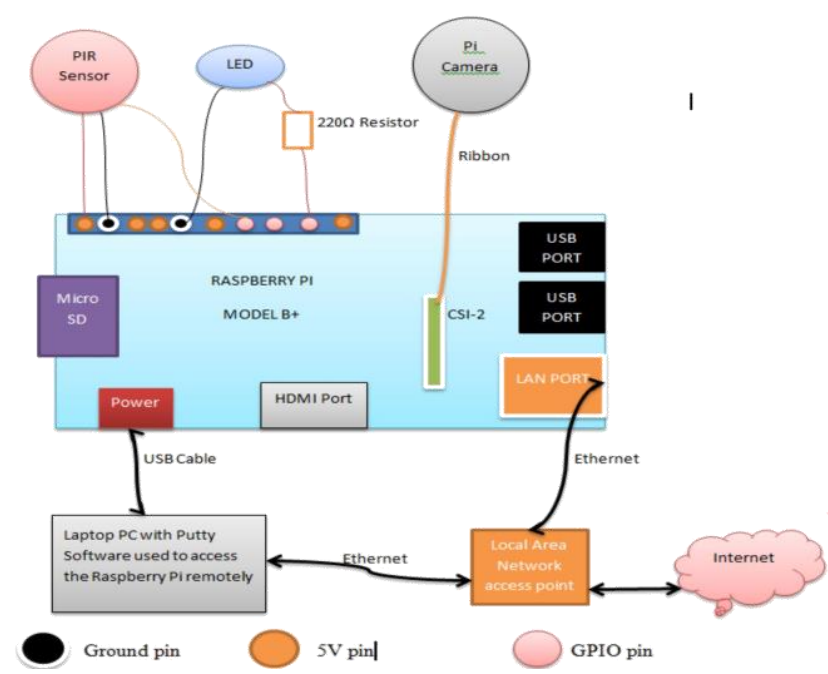

Figure 2. Hardware architecture of the security systems based on Raspberry-Pi

\subsection{Software design}

\subsubsection{The flowchart of the Raspberry-Pi Based security system}

The flowchart shown in Figure 3 is used to design and document the security systems. It illustrates the series of events starting from intrusion event up to the point when it sends out an alert. This algorithm is implemented using a Python script.

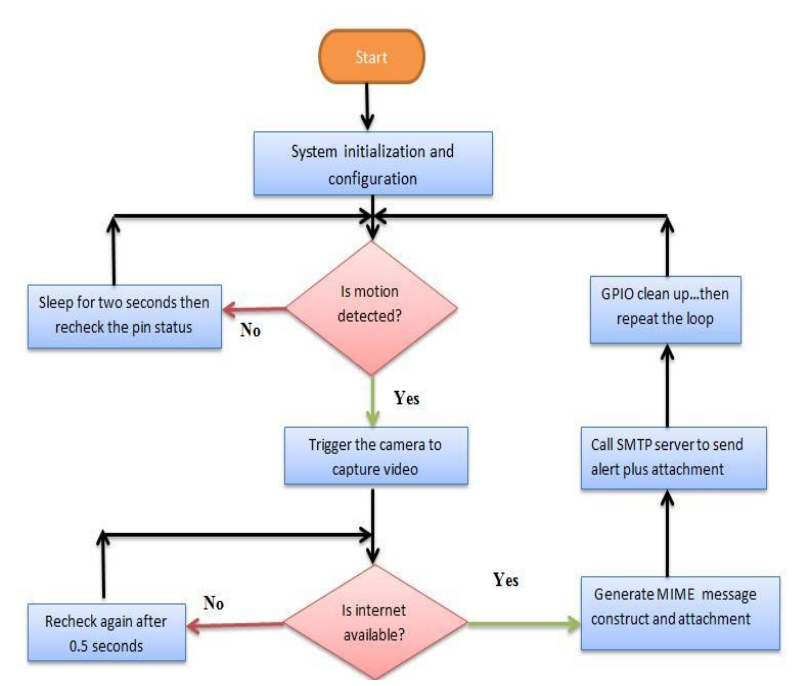

Figure 3. Flowchart implementation of the security system

\subsubsection{System initialization and configuration}

This involved the following tasks:

1 - Importing Python libraries and packages: These libraries are predefined and help in making the interfaced modules work properly.

2 - Pi Camera setting and configuration.

3 - GPIO settings and pin initialization: The channel is set using the Broadcom (BCM) channel numbering. Passive infrared pin channel is set to read mode while the led channel is set to drive/write mode.

a - Read a channel. In order to read the value of any GPIO pin, simply type; GPIO.input (channel)

b - Drive a channel. In order to drive a channel of GPIO pin, type; GPIO.output (channel, status)

This sequence of events can be elaborated well using the block diagram shown in Figure 4.

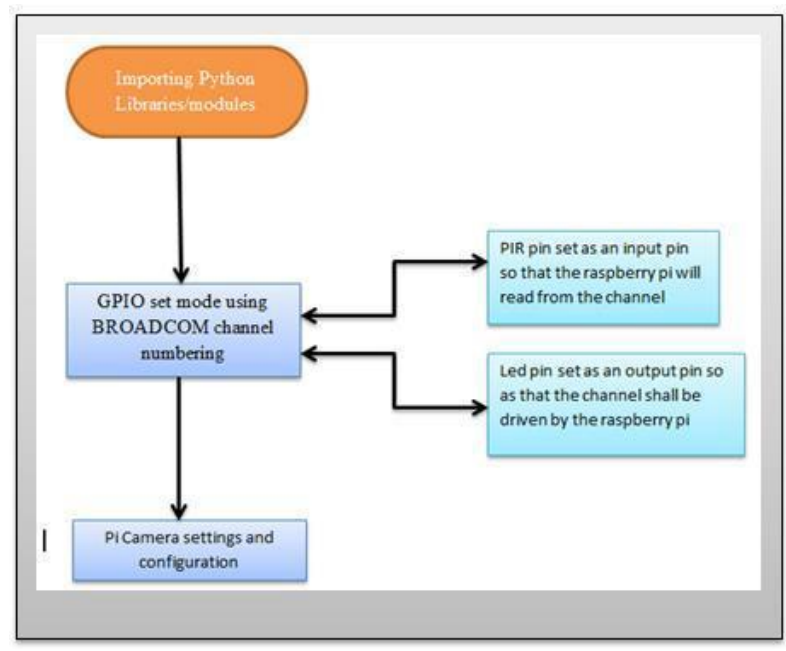

Figure 4. System initialization and configuration 


\subsubsection{Generating and sending e-mail}

After configuring the system to send an alert to the predefined subscriber, it is then necessary to generate and send the mail. Multipurpose internet mail extension (MIME) package is then called and used to generate the attachment. MIME supports characters other than ASCII, non-text attachments (audio, video and application programs) etc. It thus extends the format of an email. Simple mail transfer protocol (SMTP) program is then used to deliver the email from the Raspberry-Pi to the configured mail-hub.

\subsubsection{Pseudocode}

1. Upon restart of the system, send out email with boot IP assigned to a mail-host.

2. Check the status of the GPIO pin. If the pin is LOW, GPIO output pin 13 should remain LOW and the system is idle. Else if the pin suddenly goes HIGH. Interpret this as an interrupt event.

3. While the value of the input GPIO pin is HIGH (interrupt event), set pin 13 to be HIGH. This instance blinks the LED. Call the function that starts the Pi Camera.

4. Camera takes a 10 seconds video and save it in a file.

5. The system checks whether the internet is enabled on the Raspberry-Pi.

6. If internet, send email to a prescribed mail-host. If no internet, wait for 5 seconds then check again.

Reset the PIR sensor pin to LOW and recheck again the status after 2 seconds. This should return the program to the main loop.

\subsubsection{Developing the full code listing.}

To be able to develop the Python script that executes the algorithm defined in the flowchart, the following are done at the CLI of the Raspberry-Pi:

1 - The Pi is started, and a directory is created using mkdir command

2 - Inside the directory, a file is created using the touch command and made executable using sudo chmod $+x$ (filename).

3 - The nano command is then used to open the editor and the full Python code is written there. The script is executed using the following command:

\section{Sudo python filename.py}

\subsection{OpenCv - Python video processing}

OpenCv is a very powerful tool used to analyse images and video files. The basic processing procedure to be followed is detailed in the flowchart shown in Figure 5. Thresholding as a technique of image processing is chosen for the implementation of motion detection and tracking in video streams. The choice to script using OpenCv - Python is because Python on its own does not support video processing. There is so far no video processing library in Python. OpenCv thus provided the necessary platform to achieve image processing.

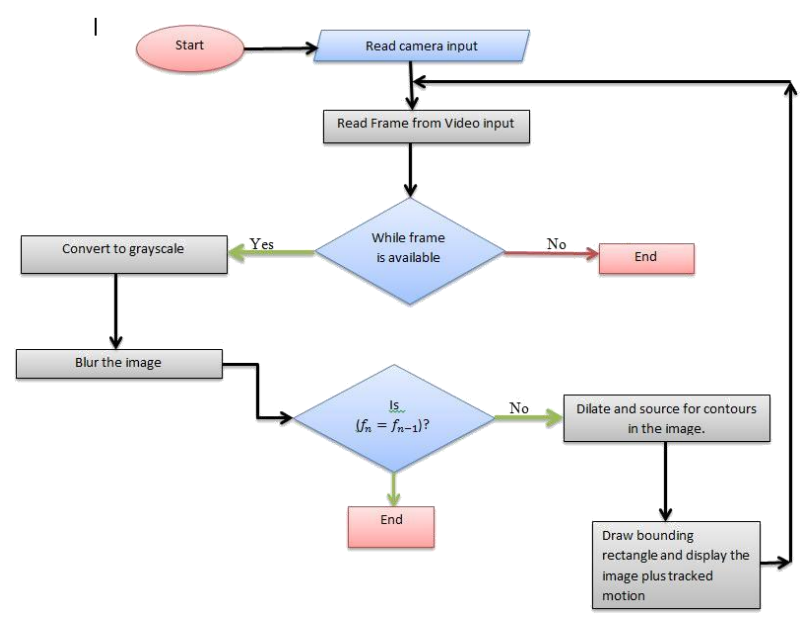

Figure 5. Flowchart of motion detection and tracking algorithm

\subsubsection{Pseudocode}

1. Start the camera and set to capture video stream.

2. Grab a frame from the video stream. If frame is grabbed, continue with the process. Else stop. Initialize the frame as the current frame.

3. Convert the captured frame to grayscale. Then do Gaussian blurring to remove noise in the gray image.

4. Capture another frame and repeat step two above.

5. Check for pixel threshold if enough to call motion detected.

6. Draw a rectangle around the region where motion is detected.

\section{RESULTS}

\subsection{Detection using a PIR sensor}

The executing of the Python script successfully yielded the results indicated in Tables 1-3. It could be shown that the variety of detection of the PIR sensor varies at distinct sensitivity stages. The better the sensitivity of the sensor the higher the variety of detection. Also, from the outcomes in Tables 1-3, it can be correctly said that the PIR sensor shaped the primary movement detector and as a result the first line of protection. All the other modules solely depended on the PIR GPIO pin to go HIGH. In case this sensor fails, the whole security procedure as designed shall fail.

Table 1. Maximum sensitivity of the PIR sensor

\begin{tabular}{|c|c|c|}
\hline Distance in meter & LED ON/OFF & Alert send \\
\hline 15 & OFF & NO \\
\hline
\end{tabular}




\begin{tabular}{|c|c|c|}
\hline 10 & OFF & NO \\
\hline 8 & OFF & NO \\
\hline 6 & YES & YES \\
\hline 5 & YES & YES \\
\hline 4 & YES & YES \\
\hline 3 & YES & YES \\
\hline
\end{tabular}

Table 2. Medium sensitivity of the PIR sensor

\begin{tabular}{|c|c|c|}
\hline Distance in meter & LED ON/OFF & Alert send \\
\hline 15 & OFF & NO \\
\hline 10 & OFF & NO \\
\hline 8 & OFF & NO \\
\hline 6 & OFF & NO \\
\hline 5 & ON & NO \\
\hline 4 & ON & YES \\
\hline 3 & ON & YES \\
\hline
\end{tabular}

Table 3. Low sensitivity of the PIR sensor

\begin{tabular}{|c|c|c|}
\hline Distance in meter & LED ON/OFF & Alert send \\
\hline 15 & OFF & NO \\
\hline 10 & OFF & NO \\
\hline 8 & OFF & NO \\
\hline 6 & OFF & NO \\
\hline 5 & OFF & NO \\
\hline 4 & OFF & NO \\
\hline 3 & ON & YES \\
\hline
\end{tabular}

\subsection{Detection and tracking using camera}

The running of the Python-OpenCv code did not yield successful results in the RPI. This is because a large video file or otherwise live video streams require large processor speed. The RPI runs at $700 \mathrm{MHz}$. This script is thus implemented differently on the laptop PC. The results in Table 4 are obtained.

From results in Table 4, it may be concluded that changing the camera pixel threshold achieves the detection and tracking action. However, detection takes place only within some limits. This can be shown by comparing the captions indicated in Figures 6-8. At a pixel threshold $=25$, motion detection and tracking are achieved, while, at 100 pixels, no detection nor tracking is possible.

Table 4. Changing the threshold level of the camera

\begin{tabular}{|l|l|l|l|l|l|l|l|l|l|}
\hline $\begin{array}{l}\text { Pixel } \\
\text { threshold }\end{array}$ & 0 & 5 & 10 & 20 & 25 & 30 & 50 & 80 & 100 \\
\hline $\begin{array}{l}\text { Motion } \\
\text { detection }\end{array}$ & NO & YES & YES & YES & YES & YES & YES & YES & NO \\
\hline Tracking & NO & YES & YES & YES & YES & YES & YES & YES & NO \\
\hline
\end{tabular}

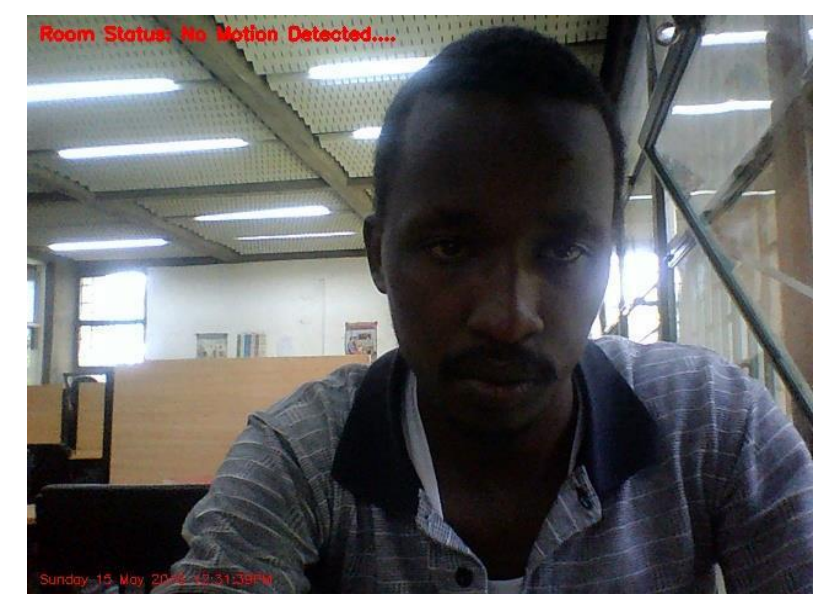

Figure 6. Motion detection and tracking in real-time video stream when the object in the field of view of the camera is stationary ( Threshold level=25 pixels)

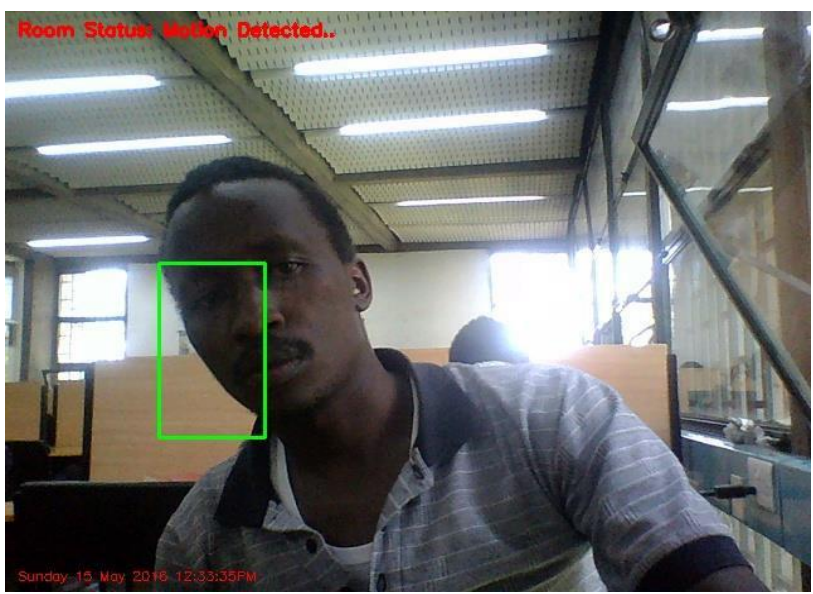

Figure 7. Tracking upon motion of an originally stationary object ( Threshold level=25 pixels)

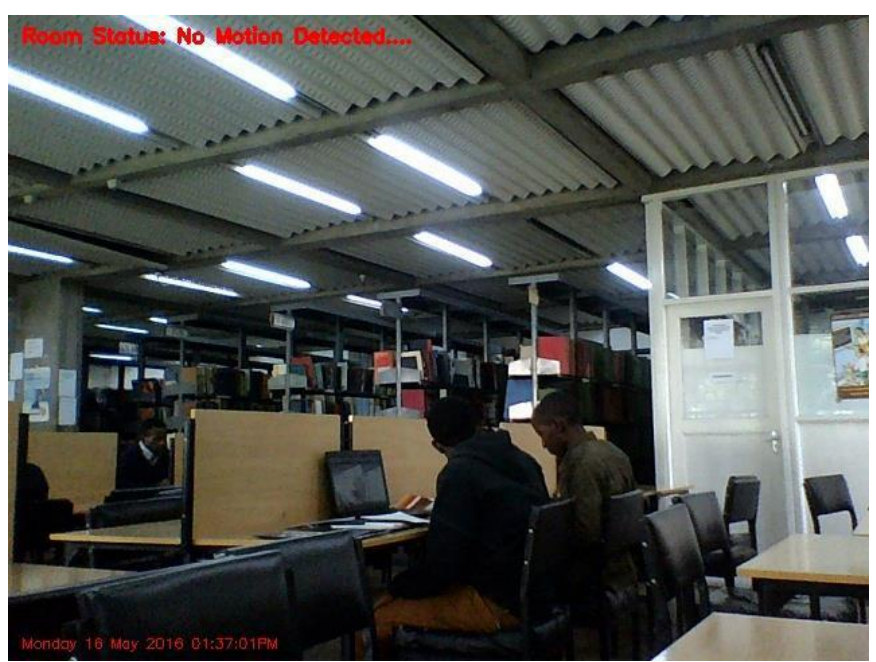

Figure 8. Detection and tracking at a threshold level of $\mathbf{1 0 0}$ pixels irrespective of object motion

\section{CONCLUSION}

This paper dealt with the design and implementation of a security system based on the Raspberry-Pi. The system aspects are motion detection using a PIR sensor, video capturing using a Pi camera and sending out an alert 
through e-mail. It did not however achieve the option of image processing in the Raspberry-Pi because of system constraints i.e. processor speed.

\section{REFERENCES}

[1] S. Sheshai, "Raspberry-Pi based security system", Graduation Project, University of Nairobi, Kenya, 2016.

[2] S. Mane, "Raspberry-Pi based security system on IoT platform", International Conference on Recent Trends in Engineering Science and Technology, Vol.5 Issue 1, PP.17-20, 2017.

[3] P. Ukey, "Development of smart home security system using Raspberry-Pi", International Research Journal of Engineering and Technology, Vol.4, Issue 6, PP.1925-1928, 2017.

[4] D. Aishwarya, "Enhanced home security using IOT and Raspberry-Pi", International Research Journal of Engineering and Technology, Vol.4, Issue 4, PP.3155-3158, 2017.

[5] S. Kavitha, S. Kamali, and P. Vijayalakshmi, "Military based security system using RaspberryPi", International Journal of Engineering and computer science, Vol.7, No.3, PP.23722-23726, 2018 . 